\title{
Significance of liver dysfunction associated with decreased hepatic CT attenuation values in Japanese patients with severe COVID-19
}

\author{
Yoshihito Uchida $^{1}$ Hayato Uemura ${ }^{1} \cdot$ Shinpei Yamaba $^{1}$ Daisuke Hamada ${ }^{1}$. \\ Norihito Tarumoto ${ }^{2} \cdot$ Shigefumi Maesaki ${ }^{2} \cdot$ Satoshi Mochida $^{1}$
}

Received: 24 June 2020/Accepted: 31 July 2020/Published online: 9 August 2020

(C) Japanese Society of Gastroenterology 2020

\begin{abstract}
Background COVID-19 has emerged as a threat to human health. Liver dysfunction has been reported to occur frequently in patients with COVID-19, although its significance has not yet been elucidated.

Methods The subjects were 35 patients with COVID-19, and clinical characteristics were retrospectively analyzed. COVID-19 patients requiring ventilator were classified as having severe COVID-19.

Results All 35 patients were diagnosed as having mild-tomoderate COVID-19 at admission, but the severity aggravated to severe in 8 patients (22.9\%) in hospital. Hepatocellular-type liver injury, defined as elevation of the serum AST and/or ALT levels to $\geq 3$ times the ULN, was seen in 2 patients (5.7\%), and cholestasis-type liver injury, defined as elevation of the serum ALP, $\gamma$-GTP and/or total bilirubin levels to $\geq$ twice the ULN, was seen in 4 patients $(11.4 \%)$. A total of 9 patients $(25.7 \%)$ fulfilled the criteria for liver injury. The percentage of patients with liver injury was higher in patients with severe COVID-19 than in the remaining patients $(P=0.001)$. Both the hepatic CT attenuation values and the liver-to-spleen attenuation $(\mathrm{L} / \mathrm{S})$ ratios at admission were lower in the former patients than in the latter patients $(P<0.001)$. ROC curve revealed the optimal cut-off value of the L/S ratio of 1.03 for discriminating between patients with severe and non-severe
\end{abstract}

Satoshi Mochida

smochida@saitama-med.ac.jp

1 Department of Gastroenterology and Hepatology, Faculty of Medicine, Saitama Medical University, 38 Morohongo, Moroyama-cho, Iruma-Gun, Saitama 350-0495, Japan

2 Department of Infectious Disease and Infection Control, Saitama Medical University, Saitama, Japan diseases. The hepatic CT attenuation values increased at the remission phase of the disease as compared to the values at admission $(P=0.012)$.

Conclusion Liver dysfunction associated with reduced hepatic CT attenuation values correlated with the disease severity in patients with COVID-19.

Keywords COVID-19 - CT values · Liver injury · SARSCoV-2

\section{Introduction}

The novel coronavirus, severe acute respiratory syndrome coronavirus 2 (SARS-CoV-2), was first isolated in December 2019 in Wuhan, China, as the causative virus of a severe form of pneumonia which was subsequently named coronavirus disease 2019 (COVID-19) [1, 2]. Since then, the virus has spread rapidly worldwide, and more than 8 million people with confirmed infection and 400,000 deaths from COVID-19 have been reported from around the world until June 2020 [3].

Although pneumonia is the main manifestation in patients with COVID-19 and is the main culprit in patients with unfavorable outcomes, COVID-19 patients can also manifest a variety of extra-respiratory symptoms, such as muscle pain, headache, dysgeusia and/or nasty odor, anorexia and gastrointestinal symptoms, such as nausea, vomiting, abdominal pain and diarrhea. Moreover, liver function test abnormalities have been reported in 15-75\% of patients with COVID-19, with the serum alanine aminotransferase (ALT) and/or aspartate aminotransferase (AST) levels being particularly high in patients with severe disease requiring assisted ventilation [4-7]. However, the significance of liver function abnormalities in relation to 
the clinical outcome has not yet been clarified in Japanese COVID-19 patients. Thus, in the present study, we analyzed the outcomes of Japanese patients with COVID-19 according to the severity of the liver function test abnormalities, including abnormal hepatic attenuation values on $\mathrm{CT}$, seen in the patients.

\section{Patients and methods}

\section{Patients}

A total of 35 patients among 43 patients diagnosed as having COVID-19 and hospitalized between March and June 2020 at the Saitama Medical University Hospital (Moroyama, Saitama, Japan) were enrolled as the subjects of this analysis. Eight patients enrolled in the clinical trial of favipiravir were excluded from the analysis according to the direction by the pharmaceutical company. The clinical features of the patients were evaluated in relation to the outcomes, especially focusing on the results of liver function tests at admission and during the course of hospitalization and CT findings obtained at admission. This study conformed to the ethical guidelines laid down in the Declaration of Helsinki, and was conducted with the approval of the Institutional Review Board of Saitama Medical University Hospital, as study no. 20045.01. Informed consent was obtained from all the patients in the form of an opt-out consent posted on the website of Saitama Medical University Hospital (https://www.saitamamed.ac.jp/hospital/outline/irb_kouhou.html). Patients who were unwilling to provide consent for participation in the study were excluded.

\section{Diagnosis of COVID-19 and classification of the disease severity}

According to the guideline published by the National Institute of Infectious Diseases (NIID), Japan, patients were definitively diagnosed as having COVID-19 when the PCR test for SARS-CoV-2 performed on nasopharyngeal swab specimens yielded a positive result. Briefly, RNA was purified from a nasopharyngeal swab specimen using the QIAamp Viral RNA Mini Kit (Qiagen K.K., Tokyo, Japan), and SARS-CoV-2 RNA was amplified by RTqPCR targeting the N2 region using the QuantiTect Probe RT-PCR Kit (Qiagen K.K.) [8].

Patients were classified into 2 groups (severe COVID-19 and mild-to-moderate COVID-19), based on the clinical symptoms and findings of clinical examinations, including chest X-ray/CT, according to the therapeutic guideline for patients with COVID-19, second edition, published by the Ministry of Health, Labour and Welfare of Japan [9]; patients requiring intensive care (ICU admission) and/or assisted ventilation due to respiratory failure were classified as having severe COVID-19, while the remaining patients were classified as having mild-to-moderate COVID-19.

\section{Liver function testing and CT imaging}

Liver function testing was performed at admission and during the course of hospitalization; the maximum values within 14 days of admission were recorded. Liver function test abnormalities were defined as follows: serum AST $>$ $37 \mathrm{U} / \mathrm{L}$, serum ALT $>40 \mathrm{U} / \mathrm{L}$, serum alkaline phosphatase $(\mathrm{ALP})>284 \mathrm{U} / \mathrm{L}$, serum $\gamma$-glutamyl transpeptidase $\quad(\gamma-\mathrm{GTP})>73 \mathrm{U} / \mathrm{L}, \quad$ and serum total bilirubin $>1.2 \mathrm{mg} / \mathrm{dL}$, corresponding to the upper limit of the normal range (ULN) for the respective parameters in the hospital laboratory. According to a previous report, liver injury can be classified into 3 types: hepatocellulartype injury, cholestasis-type injury, and mixed-type injury [4]; patients with serum ALT and/or AST levels $\geq 3$ times the ULN were classified as showing hepatocellular-type liver injury and patients with serum ALP, $\gamma$-GTP and/or total bilirubin levels $\geq$ twice the ULN were classified as showing cholestasis-type liver injury, and patients fulfilling the criteria for both the aforementioned types of liver injury were classified as showing mixed-type liver injury. Patients judged as showing abnormal liver function test results due to drug-induced liver injury (DILI) and circulatory failure, such as ischemic liver injury and congestive hepatopathy developing during and after hypovolemic shock and cardiac shock, respectively, were excluded from the analysis.

Moreover, hepatic and splenic attenuation values were measured in Hounsfield units (HU) in set regions of interest (ROIs) in the CT images obtained at admission. Two ROIs were set in the right liver lobe and one ROI in the left liver lobe. For each ROI, the largest possible area without hepatic vascular and biliary structures was selected to measure the attenuation values of the liver parenchyma. The values for the 3 ROIs were averaged to obtain the mean hepatic attenuation value. As an internal control, the mean splenic attenuation values were also calculated by averaging the values for 3 different randomly selected ROIs. Then, the liver-to-spleen attenuation ratio (L/S ratio) was calculated using the following formula: $\mathrm{L} / \mathrm{S}$ ratio $=($ mean hepatic CT attenuation value)/(mean splenic CT attenuation value).

\section{Statistical analysis}

Categorical data were compared using the Chi-squared test or Fisher's exact test. The distributions of continuous variables were analyzed by the Mann-Whitney $U$ test, and 
the differences were assessed by Wilcoxon's signed-rank test. All tests of significance were two tailed, and $P$ values of less than 0.05 were considered as denoting statistical significance. Receiver operating characteristic (ROC) curves were drawn to determine the optimal cut-off values of the test variables for predicting the outcomes of the patients. SPSS Statistics version 25 (IBM SPSS, Tokyo, Japan) was used for all the analyses.

\section{Results}

\section{Demographic characteristics and clinical features of patients with COVID-19}

The demographic characteristics and clinical features of the 35 hospitalized patients with COVID-19 enrolled in this study are shown in Table 1 . The 35 patients consisted of 22 men $(62.9 \%)$ and 13 women $(37.1 \%)$, with a median age of 52 years (range $20-83$ years). The median interval from symptom onset to hospital admission was 7 days (range 1-19 days). Underlying diseases included hypertension in 8 patients $(22.9 \%)$, diabetes mellitus in 4 patients $(11.4 \%)$, dyslipidemia in 3 patients $(8.6 \%)$, cardiac disease in 2 patients $(5.7 \%)$, bronchial asthma in 1 patient $(2.9 \%)$, and chronic hepatitis $\mathrm{C}$ in 1 patient $(2.9 \%)$. The median body mass index (BMI) was 22.5 (16.6 to 30.4).

All 35 patients were diagnosed as having mild-to-moderate COVID-19 at admission. Of the 35 patients, however, 8 patients $(22.9 \%)$ were diagnosed as having severe COVID-19 in hospital. The median age of the patients with severe COVID-19 (58 years; range 52-67) was higher than that of the patients with mild-to-moderate COVID-19 (42 years; $20-83)(P=0.034)$. The percentage of patients with underlying hypertension was marginally higher in the group with severe disease $(50.0 \%)$ than in the group with mild-to-moderate disease $(14.8 \%)(P=0.060)$, while the median BMI was similar between the two groups. Moreover, the peripheral arterial oxygen saturation levels $(\mathrm{SpO} 2)$ under room air were lower, and the percentages of

Table 1 Demographic characteristics and clinical features of patients with COVID-19

\begin{tabular}{|c|c|c|c|c|}
\hline Number of patients (\%) & $\begin{array}{l}\text { Total } \\
n=35\end{array}$ & $\begin{array}{l}\text { None severe } \\
n=27\end{array}$ & $\begin{array}{l}\text { Severe } \\
n=8\end{array}$ & $P$ value \\
\hline $\operatorname{Age}^{\mathrm{a}}$ (years) & $52(20-83)$ & $42(20-83)$ & $58(52-67)$ & 0.034 \\
\hline Male: Female $(\%)$ & 22 (62.9): 13 (37.1) & 16 (59.3): $11(40.7)$ & $6(75.0): 2(25.0)$ & 0.680 \\
\hline Body mass index ${ }^{\mathrm{a}}\left(\mathrm{kg} / \mathrm{m}^{2}\right)$ & $22.5(16.6-30.4)$ & $22.5(16.6-26.5)$ & $23.0(19.6-30.4)$ & 0.286 \\
\hline Body temperature ${ }^{\mathrm{a}}\left({ }^{\circ} \mathrm{C}\right)$ & $37.1(36.0-39.9)$ & $36.8(36.0-39.9)$ & $38.0(37.6-39.0)$ & 0.004 \\
\hline Pulse rate ${ }^{\mathrm{a}}(/ \mathrm{min})$ & $85(59-121)$ & $82(59-119)$ & $90(77-121)$ & 0.179 \\
\hline $\mathrm{SpO}_{2}{ }^{\mathrm{a}}(\%)$ & $97(89-100)$ & $98(92-100)$ & $94(89-95)$ & $<0.001$ \\
\hline \multicolumn{5}{|l|}{ Symptoms } \\
\hline Fever: $\geq 37.5^{\circ} \mathrm{C}$ & $34(97.1)$ & $26(96.3)$ & $8(100)$ & 1.000 \\
\hline Cough & $30(85.7)$ & $22(81.5)$ & $8(100)$ & 0.315 \\
\hline Fatigue & $21(60.0)$ & $14(51.9)$ & $7(87.5)$ & 0.108 \\
\hline Diarrhea & $5(14.3)$ & $1(3.7)$ & $4(50.0)$ & 0.006 \\
\hline \multicolumn{5}{|l|}{ Underling diseases } \\
\hline Hypertension & $8(22.9)$ & $4(14.8)$ & $4(50.0)$ & 0.060 \\
\hline Diabetes mellitus & $4(11.4)$ & $3(11.1)$ & $1(12.5)$ & 1.000 \\
\hline Hyperlipidemia & $3(8.6)$ & $1(3.7)$ & $2(25.0)$ & 0.124 \\
\hline Cardiac disease & $2(5.7)$ & $1(3.7)$ & $1(12.5)$ & 0.410 \\
\hline Bronchial asthma & $1(2.9)$ & $0(0)$ & $1(12.5)$ & 0.229 \\
\hline Liver diseases & $1(2.9)$ & $0(0)$ & $1(12.5)$ & 0.229 \\
\hline Bilateral pneumonia on chest CT images & $22(62.9)$ & $14(51.9)$ & $8(100)$ & 0.015 \\
\hline \multicolumn{5}{|l|}{ Antiviral therapies } \\
\hline Favipiravir & $14(40.0)$ & $7(25.4)$ & $7(87.5)$ & $<0.001$ \\
\hline Remdesivir & $1(2.9)$ & $0(0)$ & $1(12.5)$ & \\
\hline \multicolumn{5}{|l|}{ Outcome } \\
\hline Survived & $33(94.3)$ & $27(100)$ & $6(75.0)$ & 0.047 \\
\hline Dead & $2(05.7)$ & $0(0)$ & $2(25.0)$ & \\
\hline
\end{tabular}

${ }^{a}$ Values mean medium and those in parenthesis range 
patients with diarrhea and bilateral pneumonia on CT were higher in the patient group with severe disease than in the patient group with mild-to-moderate disease $(P<0.001$, $P=0.015$, and $P=0.015$, respectively). As antiviral-drug therapy, 14 patients $(40.0 \%)$ received favipiravir regardless of the clinical trial and 1 patient $(2.9 \%)$ received remdesivir, and the percentage of patients treated with antiviral therapies was higher in the patient group with severe COVID-19 than in the patient group with mild-to-moderate disease $(P<0.001)$. Of the 8 patients with severe COVID19, 2 patients, including the single patient with underlying chronic hepatitis $\mathrm{C}$ died, despite being admitted to the intensive care unit. In contrast, all of the 27 patients (77.1\%) diagnosed as having mild-to-moderate COVID-19 were recovered and the survival rate in this group of patients was significantly higher than that in the patient group with severe COVID-19 $(P=0.047)$.

\section{Liver function test abnormalities in patients with COVID-19}

The liver function test results at admission and the maximum values recorded within 14 days of admission are shown in Tables 2 and 3. At admission, a total of 17 patients (48.6\%) exhibited liver function test abnormalities, including elevated serum levels of AST, ALT, ALP, $\gamma$ GTP, and/or total bilirubin. Although the percentage of patients showing liver function test abnormalities did not differ between the 8 patients with severe COVID and 27 patients with mild-to-moderate disease (62.5 vs. $44.4 \%$, $P=0.443$ ), the serum AST, ALT, and $\gamma$-GTP levels were higher in the patient group with severe COVID-19 (AST: median 40, range 30-132; ALT: median 34, range 21-169; $\gamma$-GTP: median 47, range 26-149) than in the patient group with mild-to-moderate disease (AST: median 28, range 16-105; ALT: median 21, range 8-126; $\gamma$-GTP: median 27, range $11-208)(P=0.013, P=0.019$, and $P=0.019$, respectively).

On the other hand, $3(8.6 \%)$ patients without abnormal liver function test results developed the liver function test abnormalities during the course of hospital admission. Thus, liver function test abnormalities were seen in a total of 20 of the 43 patients $(57.1 \%)$ during the observation period. The maximum values of the serum AST (U/L), ALT (U/L), $\gamma$-GTP (U/L), and total bilirubin levels (mg/ $\mathrm{dL})$ were higher in the patient group with severe COVID19 (AST: median 82, range 32-222; ALT: median 76, range 28-237; $\gamma$-GTP: median 145, range 25-497; total bilirubin: median 1.1, range 0.7-9.6) than in the patient group with mild-to-moderate COVID-19 (AST: median 31, range 16-198; ALT: median 29, range 8-218; $\gamma$-GTP: median 30, range 13-353; total bilirubin: median 0.6, range $0.3-1.8)(P=0.010, P=0.034, P=0.003$, and $P=0.002$, respectively). Moreover, the percentage of patients showing serum ALP levels higher than the ULN were also higher in the patient group with severe COVID-19 than in the patient group with mild-to-moderate disease (50.0 vs. $22.2 \%, P=0.009$ ).

Increase of the serum AST and ALT levels to $\geq 3$ times the ULN was seen in 4 patients $(11.4 \%)$ and 5 patients (14.3\%) patients, respectively, and increase of the serum ALP, $\gamma$-GTP, and total bilirubin levels to $\geq$ twice the ULN was seen in 4 patients (11.4\%), 6 patients $(17.1 \%)$, and 2 patients $(5.7 \%)$, respectively. Thus, a total of 9 patients

Table 2 Liver function abnormalities in patients with COVID-19

\begin{tabular}{|c|c|c|c|c|}
\hline & $\begin{array}{l}\text { Total } \\
n=35\end{array}$ & $\begin{array}{l}\text { Mild-to-moderate } \\
n=27\end{array}$ & $\begin{array}{l}\text { Severe } \\
n=8\end{array}$ & $P$ value \\
\hline $\mathrm{AST}$ at adimission (U/L) & $30(16-132)$ & $28(16-105)$ & $40(30-132)$ & 0.013 \\
\hline ALT at admission (U/L) & $29(8-169)$ & $21(8-126)$ & $34(21-169)$ & 0.019 \\
\hline ALP at admission (U/L) & $185(64-755)$ & $192(109-755)$ & $178(64-309)$ & 0.563 \\
\hline$\gamma$-GTP at admission (U/L) & $30(11-208)$ & $27(11-208)$ & $47(26-149)$ & 0.019 \\
\hline Total bilirubin at admission (mg/dL) & $0.5(0.2-1.9)$ & $0.4(0.2-1.3)$ & $0.6(0.3-1.9)$ & 0.046 \\
\hline Maximum AST (U/L) & $34(16-222)$ & $31(16-198)$ & $82(32-222)$ & 0.010 \\
\hline Maximum ALT (U/L) & $038(8-237)$ & $29(8-218)$ & $76(28-237)$ & 0.034 \\
\hline Maximum ALP (U/L) & $211(109-855)$ & $202(109-755)$ & $359(158-855)$ & 0.220 \\
\hline Maximum $\gamma$-GTP (U/L) & $37(13-497)$ & $30(13-353)$ & $145(25-497)$ & 0.003 \\
\hline Maximum total bilirubin $(\mathrm{mg} / \mathrm{dL})$ & $0.7(0.3-9.6)$ & $0.6(0.3-1.8)$ & $1.1(0.7-9.6)$ & 0.002 \\
\hline Hepatic CT values (HU) & $52.55(24.07-70.85)$ & $56.09(39.07-70.85)$ & $36.47(24.07-51.08)$ & $<0.001$ \\
\hline $\mathrm{L} / \mathrm{S}$ ratio $^{\mathrm{a}}$ & $1.16(0.49-1.53)$ & $1.23(0.83-1.54)$ & $0.81(0.49-1.41)$ & $<0.001$ \\
\hline
\end{tabular}

Values mean medium and those in parenthesis range

${ }^{\text {a }}$ The liver-to-spleen attenuation rate 
Table 3 Extent of liver function abnormalities and types of liver dysfunction in patients with COVID-19

\begin{tabular}{l} 
Number of patients (\%) \\
\hline AST at admission \\
Normal \\
$1-3$ ULN \\
$>3$ ULN \\
ALT at admission \\
Normal \\
$1-3$ ULN \\
$>3$ ULN \\
ALP at admission \\
Normal \\
$1-2$ ULN \\
$>2$ ULN \\
$\gamma$-GTP at admission \\
Normal \\
$1-2$ ULN \\
$>2$ ULN
\end{tabular}

Total bilirubin at admission

Normal

1-2 ULN

$>2$ ULN

Maximum AST

$$
\begin{gathered}
\text { Normal } \\
1-3 \text { ULN } \\
>3 \text { ULN }
\end{gathered}
$$

Maximum ALT

Normal

1-3 ULN

$>3$ ULN

Maximum ALP

Normal

1-2 ULN

$>2$ ULN

Maximum $\gamma$-GTP

Normal

1-2 ULN

$>2$ ULN

Maximum total bilirubin

$$
\begin{gathered}
\text { Normal } \\
1-2 \text { ULN } \\
>2 \text { ULN }
\end{gathered}
$$

Liver test abnormality at admission

Liver test abnormality during admission

Liver injuries during admission

\begin{tabular}{|c|c|c|c|}
\hline $24(68.6)$ & $20(74.1)$ & $4(50.0)$ & 0.123 \\
\hline $10(28.6)$ & 07 (25.9) & $3(37.5)$ & \\
\hline $1(2.9)$ & $0(0)$ & $1(12.5)$ & \\
\hline $25(71.4)$ & $20(74.1)$ & $5(62.5)$ & 0.806 \\
\hline $7(20.0)$ & $5(18.5)$ & $2(25.0)$ & \\
\hline $3(8.6)$ & $2(7.4)$ & $1(12.5)$ & \\
\hline 30 (85.7) & $23(85.2)$ & 7 (87.5) & 0.676 \\
\hline $3(8.6)$ & $2(7.4)$ & $1(12.5)$ & \\
\hline $2(5.7)$ & $2(7.4)$ & $0(0)$ & \\
\hline 31 (88.6) & $26(96.3)$ & $5(62.5)$ & 0.015 \\
\hline $2(5.7)$ & $1(3.7)$ & $1(12.5)$ & \\
\hline $2(5.7)$ & $0(0)$ & $2(25.0)$ & \\
\hline 33 (94.3) & $26(96.3)$ & 7 (87.5) & 0.410 \\
\hline $2(5.7)$ & $1(3.7)$ & $1(12.5)$ & \\
\hline $0(0)$ & $0(0)$ & $0(0)$ & \\
\hline $19(54.3)$ & $17(63.0)$ & $2(25.0)$ & 0.020 \\
\hline $12(34.3)$ & $9(33.3)$ & $3(37.5)$ & \\
\hline 4 (11.4) & $1(3.7)$ & $3(37.5)$ & \\
\hline $19(54.5)$ & $16(59.3)$ & $3(37.5)$ & 0.101 \\
\hline $11(31.4)$ & $9(33.3)$ & $2(25.0)$ & \\
\hline $5(14.3)$ & $2(7.4)$ & $3(37.5)$ & \\
\hline $25(71.4)$ & $21(77.8)$ & $4(50.0)$ & 0.031 \\
\hline $6(17.1)$ & $5(18.5)$ & $3(37.5)$ & \\
\hline $4(11.4)$ & $1(3.7)$ & $1(12.5)$ & \\
\hline $23(65.7)$ & $21(77.8)$ & $2(25.0)$ & 0.009 \\
\hline $6(17.1)$ & $4(14.8)$ & $2(25.0)$ & \\
\hline $6(17.1)$ & $2(7.4)$ & $4(50.0)$ & \\
\hline 31 (88.6) & $26(96.3)$ & $5(62.5)$ & 0.015 \\
\hline $2(5.7)$ & $0(0)$ & $1(12.5)$ & \\
\hline $2(5.7)$ & $1(3.7)$ & $2(25.0)$ & \\
\hline $17(48.6)$ & $12(44.4)$ & $5(62.5)$ & 0.443 \\
\hline $20(57.1)$ & $14(51.9)$ & $6(75.0)$ & 0.419 \\
\hline $9(25.7)$ & $3(11.1)$ & $6(75.0)$ & 0.001 \\
\hline $2(5.7)$ & $1(3.7)$ & $1(12.5)$ & 0.410 \\
\hline $4(11.4)$ & $1(3.7)$ & $3(37.5)$ & 0.030 \\
\hline $3(8.6)$ & $1(3.7)$ & $2(25.0)$ & 0.124 \\
\hline
\end{tabular}

Total

Hepatocyte damage

Cholestatic

Mixed

$\begin{array}{lll}\text { Total } & \text { Mild-to-moderate } & \text { Sev } \\ n=35 & n=27 & n=8\end{array}$


(25.7\%) fulfilled the criteria for the diagnosis of liver injury, including $2(5.7 \%)$ patients with hepatocellular-type injury, $4(11.4 \%)$ patients with cholestasis-type injury, and 3 patients $(8.6 \%)$ with mixed-type injury. The percentage of patients fulfilling the diagnostic criteria for liver injury was higher in the 8 patients with severe COVID-19 than in the remaining 27 patients with mild-to-moderate disease (75.0 vs. $11.1 \%, P=0.001$ ), and the difference was accounted for entirely by patients with cholestasis-type liver injury ( 37.5 vs. $3.7 \%, P=0.030)$.

\section{Hepatic CT attenuation values in patients with COVID-19}

The findings of $\mathrm{CT}$ at admission revealed that the hepatic $\mathrm{CT}$ attenuation values (HU) and $\mathrm{L} / \mathrm{S}$ ratios were lower in the 8 patients with severe COVID-19 (HU: median 36.47, range 24.07-51.08; L/S ratio: median 0.81, range $0.49-1.41)$ than in the remaining 27 patients with mild-tomoderate disease (HU: median 56.09, range 39.07-70.85; L/S ratio: median 1.23, range 0.83-1.54) $(P<0.001$ and $P<0.001$, respectively) (Table 2 ). ROC curve analysis identified the optimal cut-off value of the L/S ratio at admission of 1.03 to predict severe COVID-19 during hospitalization, which yielded an AUC of 0.875 (sensitivity $77.8 \%$, specificity $62.5 \%, P=0.001$ ) (Fig. 1). Moreover, the $\mathrm{CT}$ attenuation values and $\mathrm{L} / \mathrm{S}$ ratios were lower in the 9 patients who fulfilled the diagnostic criteria for liver injury (HU: median 39.67, range 24.07-67.88; L/S ratio: median 0.83 , range $0.49-1.25$ ) than in the 26 patients who did not fulfill the diagnostic criteria for liver injury (HU: median 54.92, range 39.07-70.85; L/S ratio: median 1.23, range $0.87-1.54)(P=0.010$ and $P=0.002$, respectively); these differences were seen for both patients with cholestasis-type liver injury (HU: median 49.48, range 31.82-51.08; $P=0.042 ; \mathrm{L} / \mathrm{S}$ ratio: median 1.07 , range $0.64-1.15 ; P=0.035)$ and those with hepatocellular-type liver injury (HU: median 31.87, range 24.07-39.67; $P=0.040 ; \mathrm{L} / \mathrm{S}$ ratio: median 0.66 , range $0.49-0.83$; $P=0.020)$.

Of the 35 patients, the CT was repeated during the remission phase of the disease (median interval after admission, 26 days (range 19-72) in 8 patients, including 5 patients who had been diagnosed as having severe COVID19. The hepatic CT attenuation values (HU) increased from a median of 41.78 (range 24.07-69.79) at admission to 55.56 (range 37.15-70.07) in the repeat CT obtained during the remission phase $(P=0.012)$, accompanied by an increase of the L/S ratio from 0.98 (range $0.49-1.47$ ) to 1.26 (range 0.81-1.50) $(P=0.012)$ (Fig. 2).

Representative hepatic CT images in a 52-year-old woman with severe COVID-19 were shown in Fig. 3. She was diagnosed as having moderate COVID-19 at
Sensitivity

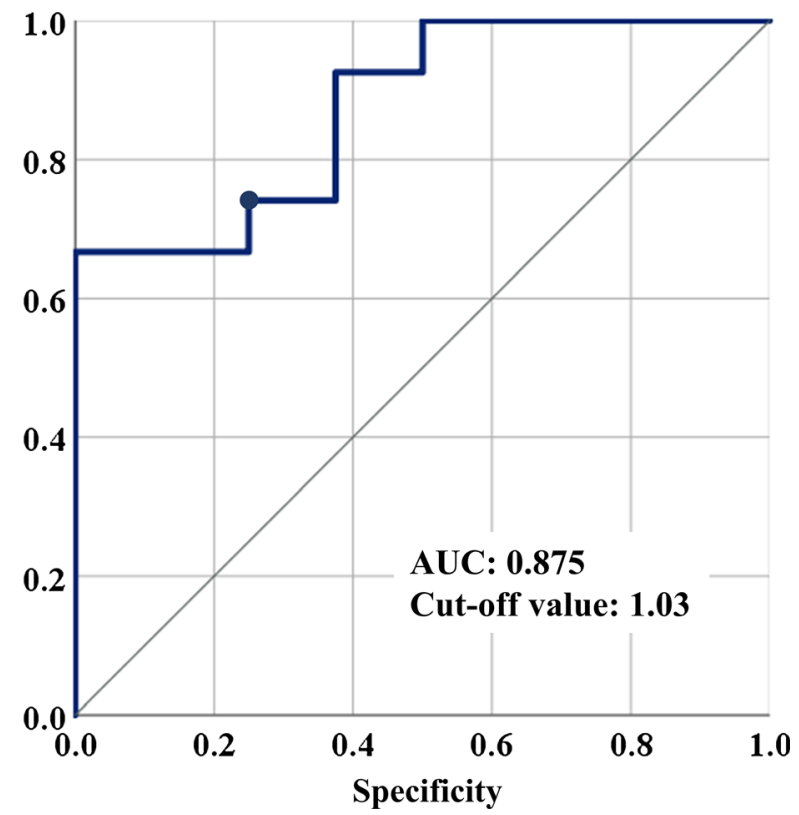

Fig. 1 Receiver operating characteristic curve for the L/S rations in CT Imaging at admission. A dot indicates the optimal cut-off points, that is, the values associated with the highest sensitivity while also maintaining the highest specificity

admission, and CT examination revealed hepatic CT attenuation values and L/S ratio as $24.07 \mathrm{HU}$ and 0.49 , respectively. She was diagnosed as having severe COVID194 days later, but was rescued following intensive therapies including intravenous infusions of high dose corticosteroid. Although body weights were increased from $59.5 \mathrm{~kg}$ at admission to $62.0 \mathrm{~kg}$ at the remission phase, both hepatic $\mathrm{CT}$ attenuation values and $\mathrm{L} / \mathrm{S}$ ratio were increased to $52.37 \mathrm{HU}$ and 1.18 , respectively, at 30 days after the admission.

\section{Discussion}

In the present study, we analyzed the clinical characteristics of 35 hospitalized patients with COVID-19, and found that of the 35, $20(57.1 \%)$ patients exhibited liver function test abnormalities, namely, elevated serum levels of AST, ALT, ALP, $\gamma$-GTP, and/or total bilirubin. The abnormalities were already detectable at admission in 17 patients (48.6\%), while they developed during the course of hospital admission in the remaining 3 patients $(8.6 \%)$. Among these patients, $9(25.7 \%)$ patients fulfilled the diagnostic criteria for liver injury, defined as elevation of the serum AST and/or ALT levels to $\geq 3$ times the UNL (hepatocellular-type liver injury) and/or elevation of the serum ALP, $\gamma$-GTP and/or total bilirubin levels to $\geq$ twice the 
Fig. 2 CT attenuation values of the liver in patients with COVID-19 in whom the CT evaluation was performed both at admission and during the remission phase. a Hepatic CT values calculated as the mean of the values in 3 different regionsof-interest (ROIs); b liver-tospleen attenuation ratios $(\mathrm{L} / \mathrm{S}$ ratios)

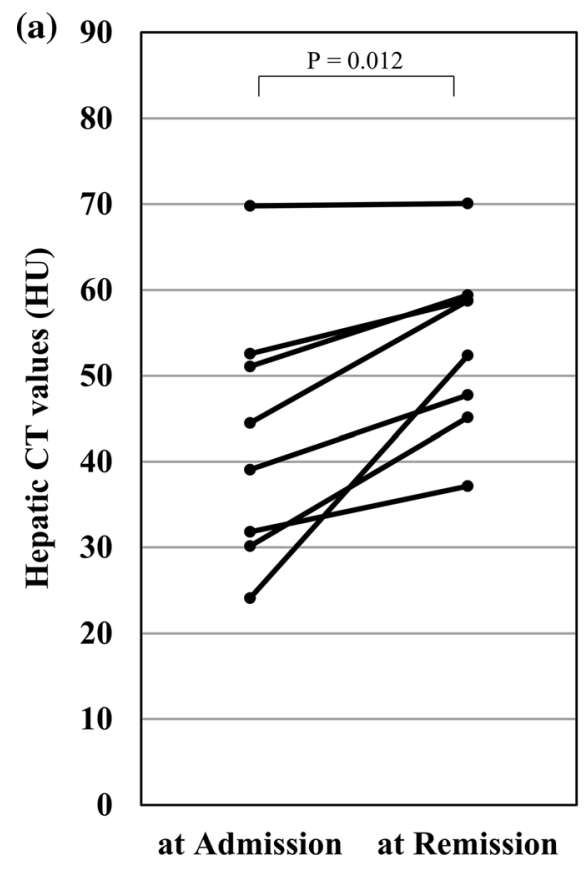

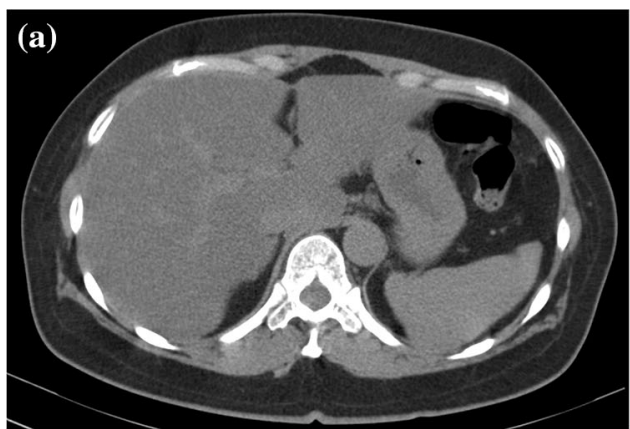

Fig. 3 Representative hepatic CT imaging in a 52-year-old woman with severe COVID-19. a CT imaging at admission. The hepatic CT attenuation value and liver-to-spleen attenuation ratio (L/S ratio) were

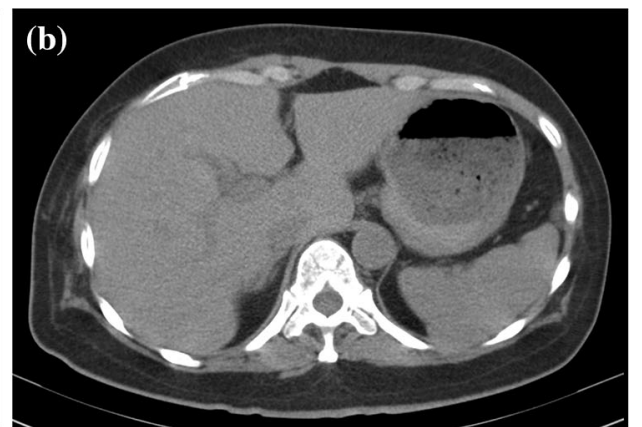

24.07 $\mathrm{HU}$ and 0.49 , respectively. b CT imaging at 30 days after admission (remission phase). The hepatic $\mathrm{CT}$ attenuation value and $\mathrm{L} / \mathrm{S}$ ratio were $52.37 \mathrm{HU}$ and 1.18 , respectively

ALT levels, and that 307 (16.7\%) out of 1,841 patients covered by 10 reports showed abnormally elevated serum total bilirubin levels [5]. Another meta-analysis conducted by Mao et al. revealed liver function test abnormalities in 241 (19.0\%) of 1,267 patients covered by 12 reports [6]. Richardson et al. reported, based on the data from a large cohort in New York city, that among 2,634 patients with COVID-19 who had already died or been discharged, evidence of acute liver injury, defined as serum AST or ALT levels $\geq 15$ times the ULN, was observed in 56 patients (2.1\%), of which 53 died (94.6\%) [10]. The frequencies of liver function test abnormalities and liver injury, especially fatal liver injury, in COVID-19 patients in Japan needs to be investigated in a large cohort in the future.

Of the 35 hospitalized patients with COVID-19, 8 patients $(22.9 \%)$ were diagnosed as having severe disease in hospital, requiring ICU monitoring and/or assisted 
ventilator, and the serum AST, ALT, and $\gamma$-GTP levels at admission were higher in the patient group with severe COVID-19 than in the patient group with mild-to-moderate disease. Moreover, the maximum serum AST, ALT, $\gamma$ GTP, and total bilirubin levels recorded within 14 days of admission were also higher in the patient group with severe disease than in the patient group with non-severe disease. Although the median serum ALP levels were similar between the two groups of patients, the percentage of patients with elevation of the serum ALP levels over the UNL during the period of admission was higher in the patient group with severe disease than in the patient group with mild-to-moderate disease. Furthermore, the percentage of patients fulfilling the diagnostic criteria for liver injury was higher in the patient group with severe COVID19 than in the patient group with mild-to-moderate disease. These data suggest that liver function test abnormalities, including those fulfilling the diagnostic criteria for liver injury, were more frequent in the patient group with severe COVID-19 than in the patient group with mild-to-moderate disease. Similar results were reported by Cai et al. [4], but they concluded that hepatocellular-type and mixed-type liver injuries were predictive of severe disease in patients with COVID-19. In contrast, in the present study, the percentage of patients with cholestasis-type liver injury was higher in the patient group with severe COVID-19 than in the patient group with mild-to-moderate disease. This discrepancy in the results between Japan and China needs to be investigated further in a multicenter study.

The mechanisms underlying the development of liver injuries in patients with COVID-19 have not yet been clarified, but it appears that SARS-CoV-2 can induce liver injury both directly and indirectly via the excessive immune responses elicited by it [11]. Angiotensin-converting enzyme-2 (ACE2) receptor has been shown to be a key receptor, expressed in various cells of the body, for SARS-CoV-2 attachment [12,13], and it is known to be expressed at a low level in the liver [14]. Wang et al. demonstrated, by electron-microscopic examination, the presence of coronavirus particles, characterized by a spike structure, in the cytoplasm of hepatocytes in patients with COVID-19; they also reported that hepatocytes in COVID19 patients showed an apoptotic binuclear appearance on histological examination [15]. Moreover, SARS-CoV-2 has been demonstrated to bind to ACE2-positive cholangiocytes, leading to damage of the cells [16]. Thus, SARSCoV-2 could directly induce both hepatocellular-type liver injury and cholestasis-type liver injury.

In the present study, only one of the patients had an underlying liver disease. The patient had been previously diagnosed as having chronic hepatitis $\mathrm{C}$, and eventually died of severe COVID-19. Based on an analysis using the linked electronic health records of 17 million adult NHS patients in England, Williamson et al. identified underlying chronic liver disease as a risk factor for COVID-19-related hospital death, with a hazard ratio of 1.61 [17]. Similar results were reported by Singh et al., based on analysis of data obtained from electronic health records in the United States; they reported pre-existing liver disease as a risk factor for death in patients with COVID-19, with a hazard ratio of 2.8 [18]. According to a report from China, among various liver diseases, non-alcoholic liver disease (NAFLD) was identified as a risk factor for progression of COVID-19 in 202 patients [19]. The significance of underlying hepatitis B virus (HBV) infection and hepatitis $\mathrm{C}$ virus (HCV) infection still remains to be elucidated $[20,21]$. Therefore, further investigations in the Japanese population are needed.

To our surprise, hepatic CT findings at admission were associated with the disease severity of patients with COVID-19 during the hospitalization. Both the hepatic CT attenuation values and the L/S ratios were lower in the patient group with severe COVID-19 than in the patient group with mild-to-moderate COVID-19 despite that both patients were diagnosed as mild-to-moderate COVID-19 at admission when CT examinations were done. Moreover, the hepatic CT attenuation values were higher during the remission phase as compared to the values at admission. In general, hepatic CT attenuation values are reduced in patients with steatosis, and in patients with acute liver failure, necrotic areas in the liver have been reported to show decreased CT attenuation values [22]. The median BMI and percentages of patients with underling diabetes were similar between the patient group with severe COVID-19 and the patient group with mild-to-moderate COVID-19, as well as between patients with and without evidence of liver injury in our study, suggesting that factors other than baseline hepatic steatosis may contribute to the reduced hepatic $\mathrm{CT}$ attenuation values in the patients with severe COVID-19 observed in this study. Moreover, considering that both patients with cholestasis-type liver injury and patients with hepatocellular-type liver injury showed reduced hepatic $\mathrm{CT}$ attenuation values, the contribution of liver necrosis to the decreased hepatic $\mathrm{CT}$ attenuation values seen in patients with severe COVID-19 seems unlikely. The contribution of hepatic steatosis, however, should be further investigated, since SARS-CoV-2 infection was shown to affect lipid metabolisms through decrease of serum LDL and HDL levels especially in patients with severe COVID-19 [23, 24]. The reasons for the decreased hepatic CT attenuation values in patients with severe COVID-19 need to be investigated in the future in reference to hepatic CT attenuation values in patients with other hepatotoxic viral infection. ROC analysis, however, revealed an optimal cut-off value of the L/S ratio in the CT obtained at admission of 1.03 , to identify patients 
with severe COVID-19. The L/S ratio in the CT obtained at admission merits investigation as a potential marker to predict an unfavorable outcome in patients with COVID19.

The present study was done retrospectively in a single institute subjected for a small number cohort, and we had to exclude 8 patients enrolled in the clinical trial of favipiravir under direction by the Good Clinical Practice (GCP) in Japan. The former limitation should be overcome in a large number cohort enrolled in multiple institutions in the future. Biases due to the latter matter, however, would be excluded in the present study, since the same results were obtained when analyses were done in a total of 43 patients including 8 patients receiving favipiravir (data not shown). Moreover, underling liver diseases including hepatic steatosis preceding SARS-CoV-2 infection were unclear in the most patients in the present study. Patients with lean-type NASH [25] might be present in those manifesting decreased CT attenuation values in the liver. This matter should be also solved in the further prospective study.

In conclusion, liver function test abnormalities, especially evidence of cholestasis-type liver injury, were more frequent in the patient group with severe COVID-19 than in the patient group with mild-to-moderate COVID-19. Moreover, the hepatic CT attenuation values as well as the $\mathrm{L} / \mathrm{S}$ ratios were lower in the patient group with severe COVID-19 than in the patient group with mild-to-moderate disease. Future studies in larger cohorts are warranted to precisely clarify the significance of liver injury associated with low hepatic CT attenuation values in Japanese patients with COVID-19.

\section{References}

1. Huang C, Wang Y, Li X, et al. Clinical features of patients infected with 2019 novel coronavirus in Wuhan, China. Lancet. 2020;395:497-506.

2. Li Q, Guan X, Wu P, et al. Early transmission dynamics in Wuhan, China, of novel coronavirus-infected pneumonia. N Engl J Med. 2020;382:1199-207.

3. World Health Organization (WHO) Coronavirus disease (COVID-2019) situation reports. https://www.who.int/emergen cies/diseases/novel-coronavirus-2019/situation-reports

4. Cai Q, Huang D, Yu H. COVID-19: abnormal liver function tests. J Hepatol. 2020. https://doi.org/10.1016/j.jhep.2020.04.006.

5. Sultan S, Altayar O, Siddique SM, et al. AGA Institute Rapid Review of the Gastrointestinal and Liver Manifestations of COVID-19, Meta-analysis of international data, and recommendations for the consultative management of patients with COVID19. Gastroenterology. 2020;159:320.e27-34.e27.

6. Mao R, Qiu Y, He J, et al. Manifestations and prognosis of gastrointestinal and liver involvement in patients with COVID19: a systematic review and meta-analysis. Lancet Gastroenterol Hepatol. 2020;5:667-78.
7. Qi X, Liu Y, Wang J, et al. Clinical course and risk factors for mortality of COVID-19 patients with pre-existing cirrhosis: a multicentre cohort study. Gut. 2020. https://doi.org/10.1136/ gutjnl-2020-321666.

8. Shirato K, Nao N, Katano H, et al. Development of genetic diagnostic methods for novel coronavirus 2019 (nCoV-2019) in Japan. Jpn J Infect Dis. 2020. https://doi.org/10.7883/yoken.JJID. 2020.061.

9. The therapeutic guideline for patients with COVID-19, second edition, published by the Ministry of Health, Labour and Welfare of Japan. https://www.mhlw.go.jp/content/000631552.pdf. Accessed 24 June, 2020.

10. Richardson S, Hirsch JS, Narasimhan M, et al. Presenting characteristics, comorbidities, and outcomes among 5700 patients hospitalized with COVID-19 in the New York City area. JAMA. 2020;323:2052-9.

11. Sun J, Aghemo A, Forner A, et al. COVID-19 and liver disease. Liver Int. 2020;40:1278-81.

12. Hoffmann M, Kleine-Weber H, Schroeder S, et al. SARS-CoV-2 cell entry depends on ACE2 and TMPRSS2 and is blocked by a clinically proven protease inhibitor. Cell. 2020;181(271-280):e8.

13. Yan R, Zhang Y, Li Y, et al. Structural basis for the recognition of SARS-CoV-2 by full-length human ACE2. Science. 2020;367:1444-8.

14. Clarke NE, Turner AJ. Angiotensin-converting enzyme 2: the first decade. Int J Hypertens. 2012;2012:307315.

15. Wang Y, Liu S, Liu H, et al. SARS-CoV-2 infection of the liver directly contributes to hepatic impairment in patients with COVID-19. J Hepatol. 2020;S0168-8278:30294-304.

16. Chai X, Hu L, Zhang Y, et al. Specific ACE2 expression in cholangiocytes may cause liver damage after 2019-nCoV infection. bioRxiv. 2020. https://doi.org/10.1101/2020.02.03.931766.

17. The OpenSAFELY Collaborative, Williamson E, Walker AJ, et al. OpenSAFELY: factors associated with COVID-19-related hospital death in the linked electronic health records of 17 million adult NHS patients. bioRxiv. 2020. https://doi.org/10.1101/2020. 02.03.931766.

18. Singh S, Khan A. Clinical characteristics and outcomes of COVID-19 among patients with pre-existing liver disease in United States: a multi-center research network study. Gastroenterology. 2020;S0016-5085:30585.

19. Ji D, Qin E, Xu J, et al. Non-alcoholic fatty liver diseases in patients with COVID-19: a retrospective study. J Hepatol. 2020;S0168-8278:30206-133.

20. Xu L, Liu J, Lu M, et al. Liver injury during highly pathogenic human coronavirus infections. Liver Int. 2020;40:998-1004.

21. AASLD'S Clinical insights for hepatology and liver transplant providers during the COVID-19 pandemic. 2020. https://www. aasld.org/sites/default/files/2020-06/AASLD-COVID19-Expert PanelConsensusStatement-June42020-FINAL.pdf Accessed 4 June, 2020.

22. Yasui S, Fujiwara K, Okitsu K, et al. Importance of computed tomography imaging features for the diagnosis of autoimmune acute liver failure. Hepatol Res. 2012;42:42-50.

23. Wei X, Zeng W, Su J, et al. Hypolipidemia is associated with the severity of COVID-19. J Clin Lipidol. 2020;14:297-304.

24. Fan J, Wang H, Ye G, et al. Letter to the Editor: Low-density lipoprotein is a potential predictor of poor prognosis in patients with coronavirus disease 2019. Metabolism. 2020;107:154243.

25. Das K, Das K, Mukherjee PS, et al. Nonobese population in a developing country has a high prevalence of nonalcoholic fatty liver and significant liver disease. Hepatology. 2010;51:1593-602.

Publisher's Note Springer Nature remains neutral with regard to jurisdictional claims in published maps and institutional affiliations. 